\title{
Promoting a Sense of Belonging Among Underrepresented Medical Students Through Cross-Cultural Programming
}

\author{
Kaitlin Wendel ${ }^{1}$, Nabia Khan ${ }^{1}$, Antwione Haywood ${ }^{2}$ \\ ${ }^{1}$ Indiana University School of Medicine; ${ }^{2}$ Indiana University School of Medicine, Medical \\ Student Education
}

Background and Hypothesis: Despite improvements in recruiting trainees from underrepresented backgrounds, there is a paucity in programming designed to enhance underrepresented in medicine (URM) students' sense of belonging and well-being. In addition to academic stressors, URM students may experience discrimination and stereotype threat, all of which contribute to burnout. Although Indiana University School of Medicine is one of the largest and more diverse medical schools in the country, URM students continue to experience disconnectedness from peers, mentors, and faculty from URM backgrounds due to the regional campus model. To address the complex challenges URM students face, the Cross-Cultural Student Success Retreat (CCSSR) was created. We hypothesize that URM students will feel an increased sense of belonging through participation in the retreat.

Project Methods: The CCSSR was developed in partnership between Medical Student Education and the Office of Diversity Affairs as a response to concerns of isolation and burnout among URM students. The program is designed to be cohort style where day-long events take place twice an academic year to engage students with minority faculty and activities designed to promote sense of belonging, retention, and success. The retreat is broken into four sessions; self-discovery, self-efficacy, bias/perception, and well-being. Each of the sessions includes a presentation, discussion, and activity.

Results: The retreat was found to be a success, with positive responses received from 36 participants across each session. Outcomes included an increased awareness of personal biases and prejudices, heightened sense of belonging among peers, and a feeling of empowerment to make their environment more inclusive.

Conclusion and Potential Impact: The positive outcomes of the retreat strongly suggest a need to implement long and short-terms programs catering specifically to the success of URM students. Ultimately, addressing the specific challenges faced by URM students will decrease rates of burnout and isolation among this population. 